\title{
New Results on Nucleosynthesis in Massive Stars: Nuclear Data Needs for Nucleosynthesis
}

R. D. Hoffman, T. Rauscher, A. Heger, S. E. Woosley

This article was submitted to the International Conference on Nuclear Data for Science and Technology, Tsukuba, Japan, October 7-12, 20001

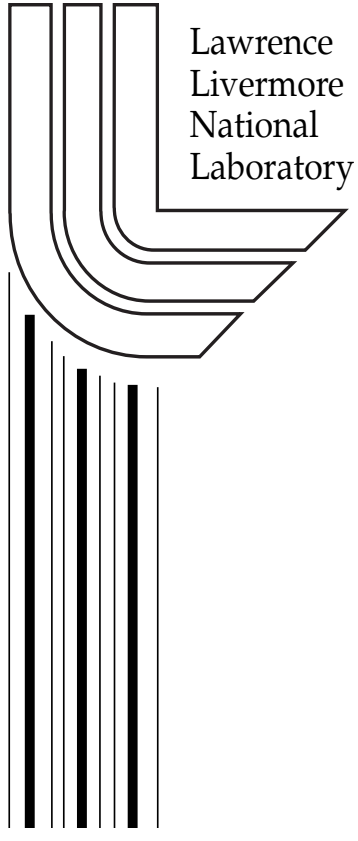

November 9, 2001 


\section{DISCLAIMER}

This document was prepared as an account of work sponsored by an agency of the United States Government. Neither the United States Government nor the University of California nor any of their employees, makes any warranty, express or implied, or assumes any legal liability or responsibility for the accuracy, completeness, or usefulness of any information, apparatus, product, or process disclosed, or represents that its use would not infringe privately owned rights. Reference herein to any specific commercial product, process, or service by trade name, trademark, manufacturer, or otherwise, does not necessarily constitute or imply its endorsement, recommendation, or favoring by the United States Government or the University of California. The views and opinions of authors expressed herein do not necessarily state or reflect those of the United States Government or the University of California, and shall not be used for advertising or product endorsement purposes.

This is a preprint of a paper intended for publication in a journal or proceedings. Since changes may be made before publication, this preprint is made available with the understanding that it will not be cited or reproduced without the permission of the author.

This work was performed under the auspices of the United States Department of Energy by the University of California, Lawrence Livermore National Laboratory under contract No. W-7405-Eng-48.

This report has been reproduced directly from the best available copy.

Available electronically at http://www.doc.gov/bridge

Available for a processing fee to U.S. Department of Energy

And its contractors in paper from

U.S. Department of Energy

Office of Scientific and Technical Information

P.O. Box 62

Oak Ridge, TN 37831-0062

Telephone: (865) 576-8401

Facsimile: (865) 576-5728

E-mail: reports@adonis.osti.gov

Available for the sale to the public from

U.S. Department of Commerce

National Technical Information Service

5285 Port Royal Road

Springfield, VA 22161

Telephone: (800) 553-6847

Facsimile: (703) 605-6900

E-mail: orders@ntis.fedworld.gov

Online ordering: http://www.ntis.gov/ordering.htm

Or

Lawrence Livermore National Laboratory

Technical Information Department's Digital Library

http://www.llnl.gov/tid/Library.html 


\title{
New Results on Nucleosynthesis in Massive Stars; Nuclear Data Needs for Nucleosynthesis
}

\author{
Robert HOFFMAN ${ }^{1, *}$, Thomas RAUSCHER ${ }^{2}$, Alex HEGER ${ }^{3}$, and Stan WOOSLEY ${ }^{3}$ \\ ${ }^{1}$ Nuclear Theory \& Modeling Group, L-414, Lawrence Livermore National Laboratory, Livermore, CA 94551, USA \\ ${ }^{2}$ Institut fuer Theoretische Physik, Universitaet Basel, Klingelbergstr. 82 CH-4056 Basel, Switzerland \\ ${ }^{3}$ Department of Astronomy \& Astrophysics, Univ. of Calif. Santa Cruz, Santa Cruz, CA 95064, USA
}

\begin{abstract}
We review the current status of the nuclear reaction rates needed to study nucleosynthesis in massive stars. Results for the calculated nucleosynthesis of all stable species from Hydrogen to Bismuth in a completely evolved $25 \mathrm{M}_{\odot}$ star of initial solar metallicity will be presented. Special emphasis will be paid to two particular reactions, ${ }^{12} \mathrm{C}(\alpha, \gamma){ }^{16} \mathrm{O}$ and ${ }^{22} \mathrm{Ne}(\alpha, n){ }^{25} \mathrm{Mg}$, and their effect on the structure of the star and resultant nucleosynthesis. Both have been measured many times, but the present range of experimental uncertainty translates into remarkable sensitivity of the calculated nucleosynthesis.
\end{abstract}

KEYWORDS: nucleosynthesis, nuclear reactions, abundances, supernovae - general

\section{Introduction}

Second only in scientific interest to the origin of the universe itself is the origin of the chemical elements therein. The near term goal of nuclear origins, or nucleosynthesis, seeks to develop a complete understanding of the origin of every isotope in nature in terms of their nuclear properties and astrophysical sites where they were assembled. Once known, this understanding can also be employed to calculate the chemical history, not only of the elements in our own sun and the Galaxy, but in older stars, future stars, and in distant galaxies.

Simulating nucleosynthesis in stars presents several problems for nuclear physics that are peculiar to the stellar environment. For many species of interest, the important nuclear flows move through target or product nuclei (sometimes both) that are frequently radioactive. Subsequently, many important reaction cross sections are not amenable to measurement. Further, neutron resonance analysis can often only be done on compound nuclear states one neutron removed from stability, and thus important quantities derived from such an analysis (average level spacings, neutron strength functions, etc.) are not available for input to reaction models on unstable nuclei. The potential for Rare Isotope Accelerators to address some of the more critical reactions is tantalizing, but due to shear numbers alone, most of our nuclear cross section needs for nucleosynthesis will necessarily be derived from theory.

Another complication is that the targets exist in a thermal distribution of excited states. Laboratory measurements (where available) cannot be directly applied to reaction networks. Further, reactions on long-lived or highly populated isomeric states are not included in stellar reaction networks used to calculate nucleosynthesis in massive stars $\left({ }^{26} \mathrm{Al}\right.$ being the notable exception).

Finally, there are a lot of nuclei for which nuclear reaction rate information is needed, roughly 4600 bound nuclei exist from hydrogen to lead. Considering only binary reactions involving nucleons, alpha-particles, and photons, along with a

\footnotetext{
${ }^{*}$ Corresponding author, Tel. +011-925-424-6411

E-mail: rdhoffman@1lnl.gov
}

potential ground state weak interaction $\left(\beta^{-}, \beta^{+}\right.$, or electron capture), this translates into approximately 32,000 nuclear reactions (plus their inverses) if one considers all possible nuclear burning scenarios. In addition, a need for weak interaction rates at extreme values of temperature and density experienced in the late time evolution of massive stars increases these nuclear data requirements. Clearly, the development of reaction rate data bases to study nucleosynthesis in massive stars is a huge undertaking, drawing from the best elements of experiment, theory, and evaluation. That such efforts have only been made three times in the last 25 years should come as no surprise. ${ }^{1-3)}$

This contribution will describe the current status of nuclear reaction rate information required to study nucleosynthesis in massive stars. Examples of nucleosynthesis in a $25 \mathrm{M}_{\odot}$ star of initial solar metallicity is given in $§ I I$. The required data will be broken into categories depending on reaction type and range of target nuclei, and their impact on the nucleosynthesis will be discussed ( $§$ III.) Sensitivity to key reaction rates will be presented for two specific cases ( $§$ III.2 and III.3). Weak rates are treated in $\S \mathrm{IV}$.

\section{Nucleosynthesis in Massive Stars}

Nucleosynthesis in stars has been reviewed many times. ${ }^{4-6)}$ To set the stage we present in Table 1 the nuclear burning stages experienced by massive stars $\left(M \geq 11 \mathrm{M}_{\odot}\right)$. Shown are the major hydrostatic burning phases $(\mathrm{H}, \mathrm{He}, \mathrm{C}, \mathrm{Ne}, \mathrm{O}$, and Si burning), their principle product(s), most important secondary products, and the approximate temperature and lifetimes during which a given fuel is burned. The general trends are evident: an abundant "fuel" burns, liberating energy which serves to support the star against gravitational contraction. Eventually the fuel is exhausted, the star contracts, the core heats up, and the "ash" of the previous burning stage serves as the "fuel" for the next one. A shell of material adjacent to the new burning core continues to burn by the same reaction mechanisms as in the previous core burning scenario, and the star develops an "onion-like" structure. To burn heavier fuels the temperature must increase to surmount the increas- 
ing coulomb charge barriers, and the lifetime for exhausting a given fuel decreases owing to: a) the very high temperature dependence of the reaction rates governing the energy release, and $b$ ) the ever mounting neutrino losses (which dominate beyond helium burning).

Table 1 Advanced Nuclear Burning Stages

\begin{tabular}{ccccc}
\hline Fuel & $\begin{array}{c}\text { Main } \\
\text { Product }\end{array}$ & $\begin{array}{c}\text { Secondary } \\
\text { Products }\end{array}$ & $\begin{array}{c}\text { Temp } \\
10^{9} \mathrm{~K}\end{array}$ & $\begin{array}{c}\tau \\
\mathrm{yr}\end{array}$ \\
\hline $\mathrm{H}$ & $\mathrm{He}$ & ${ }^{14} \mathrm{~N}$ & 0.03 & $10^{7}$ \\
$\mathrm{He}$ & $\mathrm{C}, \mathrm{O}$ & ${ }^{18} \mathrm{O},{ }^{22} \mathrm{Ne}$, & 0.2 & $10^{6}$ \\
& \multicolumn{5}{c}{$s-$ process } \\
$\mathrm{C}$ & $\mathrm{Ne}, \mathrm{Mg}$ & $\mathrm{Na}$ & 0.8 & $10^{3}$ \\
$\mathrm{Ne}$ & $\mathrm{O}, \mathrm{Mg}$ & $\mathrm{Al}, \mathrm{P}$ & 1.5 & $\sim 10^{-1}$ \\
$\mathrm{O}$ & $\mathrm{Si}, \mathrm{S}$ & $\mathrm{Cl}, \mathrm{Ar}$, & 2.0 & $\sim 2$ \\
& \multicolumn{5}{c}{$\mathrm{K}, \mathrm{Ca}$} \\
$\mathrm{Si}$ & $\mathrm{Fe}$-group & $\mathrm{Ti}, \mathrm{V}, \mathrm{Cr}$, \\
& & 3.3 & $\sim 10^{-2}$ \\
& & $\mathrm{Mn}, \mathrm{Co}, \mathrm{Ni}$ & & \\
\hline
\end{tabular}

Following core silicon-burning, the core collapses, and if a neutron star forms at the stars center (likely for stars less than $30 \mathrm{M}_{\odot}$ ), a Type II supernova explosion can occur. Ignoring the hydrodynamic and neutrino-transport issues, the outgoing shock wave heats the overlying shells of previously synthesized elements, and explosive nucleosynthesis can alter the composition of certain species. Additional nucleosynthetic processes can take place, including the $\nu$-process, ${ }^{7)}$ the $p$-process, ${ }^{8)}$ and possibly the $r$-process. ${ }^{9)}$ For a comprehensive survey of massive star evolution and nucleosynthesis, see Woosley \& Weaver. ${ }^{10)}$ Galactic chemical evolution is explored in Timmes, Woosley, \& Weaver. ${ }^{11)}$

The final nucleosynthesis (including mass loss) of a fully evolved solar metallicity $25 \mathrm{M}_{\odot}$ star $^{12)}$ is presented in Figure 1. The reaction network included all necessary isotopes through mass 210 and used reaction rates current as of 2001. Shown is the "production factor" vs. mass number. Isotopes of a given element are connected by solid lines. The dashed line is centered on ${ }^{16} \mathrm{O}$, with the dotted lines representing a "success band" of \pm 0.3 dex. With rare exception, all isotopes from oxygen through nickel are co-produced in solar proportions with a production factor of $\sim 15$ (in this figure, the initial composition of the sun would be a set of points all lying on "1"; a production factor of 15 means that the suns complement of metals could be understood if $1 / 15$ of its mass passed through conditions like those experienced in this $25 \mathrm{M}_{\odot}$ star). The $r-, s-$, and $p$-process isotopes are also well produced (perhaps a little over-produced) from nickel to about $\mathrm{A}=88$. In a $15 \mathrm{M}_{\odot}$ star, ${ }^{13)}$ the $s$-process yield is less. The yields of these "trans-iron" elements is also sensitive to a still poorly determined reaction rate for ${ }^{22} \mathrm{Ne}(\alpha, n)^{25} \mathrm{Mg}$ (see $§$ III.3). Above mass 90, nucleosynthesis in massive stars is mostly restricted to the $p$ - process, although a potential $r$-process contribution from a $\nu$-driven wind ${ }^{9)}$ is not included here.
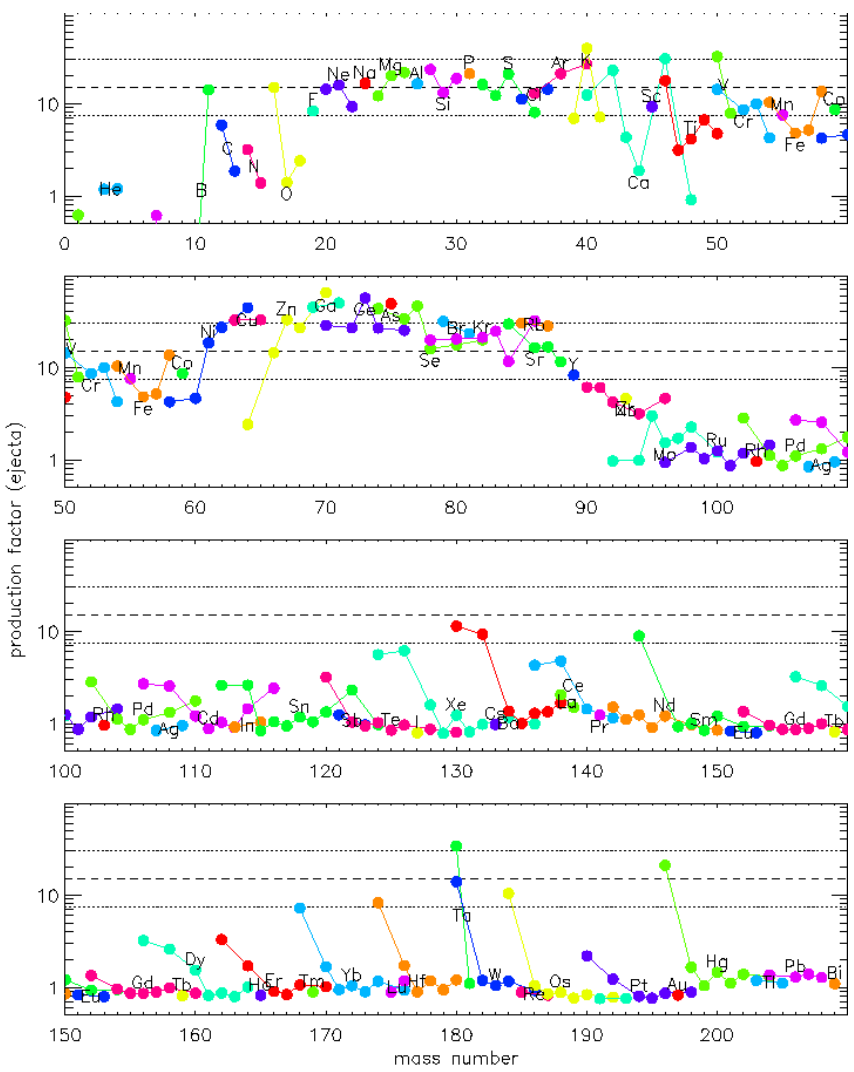

Fig. 1 Decayed, post-explosive production factors for a $25 \mathrm{M}_{\odot}$ star of initial solar metallicity.

\section{Strong and Electromagnetic Reaction Rates}

We now describe the important nuclear physics ingredients needed to calculate stellar nucleosynthesis as displayed in Figure 1. The discussion breaks nicely into two distinct regions: reactions on targets lighter than silicon (where many of the key cross sections have been measured), and reactions on heavier targets (where the community must rely on statistical theory).

\section{Critical Reactions for Energy Generation}

Along with the $3 \alpha$ reaction rate, the heavy ion reactions ${ }^{12} \mathrm{C}+{ }^{12} \mathrm{C},{ }^{12} \mathrm{C}+{ }^{16} \mathrm{O}$, and ${ }^{16} \mathrm{O}+{ }^{16} \mathrm{O}$ are the most important reactions for energy generation during hydrostatic helium, carbon, and oxygen burning. As such these reactions play a major role in determining stellar structure, and the production of all stable self-conjugate $(\mathrm{Z}=\mathrm{N})$ nuclei which constitute the most abundant species found in nature after hydrogen and helium (formed in the Big Bang). The current source for these reaction rates is Caughlan \& Fowler, ${ }^{14)}$ although the $3 \alpha$ reaction rate has recently been re-evaluated ${ }^{15)}$ (little change resulted over the temperature range at which helium burning operates). These are probably adequate with the possible exception of ${ }^{12} \mathrm{C}+{ }^{12} \mathrm{C}$ and its electron screening rate. 


\section{2. ${ }^{12} \mathbf{C}(\alpha, \gamma){ }^{16} \mathbf{O}$}

Possibly the most important nuclear burning scenario as far as nucleosynthesis is concerned is helium burning, being the only energy generation scenario in which two reactions, $3 \alpha$ and ${ }^{12} \mathrm{C}(\alpha, \gamma){ }^{16} \mathrm{O}$, compete on so nearly an equal basis in the consumption of a major fuel. It is here that a star has the ability to change its neutron excess and produce nuclei that have more neutrons than protons. In combination with $3 \alpha$, the ${ }^{12} \mathrm{C}(\alpha, \gamma)$ rate determines the ratio of carbon to oxygen at the end of core helium burning, with dramatic consequences for stellar structure, ultimately affecting the size and composition of the iron core, the possible nature of a compact remnant, and also the future of all subsequent phases of nuclear burning. Shown in Figure 2 are the variations in core mass size for the advanced burning stages in a $25 \mathrm{M}_{\odot}$ star of solar metallicity as a function of a multiplier on the ${ }^{12} \mathrm{C}(\alpha, \gamma){ }^{16} \mathrm{O}$ reaction rate of Buchmann. ${ }^{16)}$

Although considered to be the most important reaction rate, its experimental value over the temperature range of interest $(300 \mathrm{keV})$ is inadequately known. Determination of an accurate rate is experimentally challenging because it proceeds through two sub-threshold resonances whose critical alphawidths must be determined indirectly. ${ }^{16-18)}$ The rate is divided into three parts, a) the electric dipole part proceeding through the $1^{-}$resonance, $b$ ) the electric quadrapole part proceeding through the $2^{+}$resonance, and everything else. The total rate is often expressed in terms of the S-factor at 300 $\mathrm{keV}$, and the current uncertainties place its value in the range of 100 to $200 \mathrm{keV}$ barns (but with a preference for 150 to $170 \mathrm{keV} \mathrm{b}$ with a temperature dependence similar to that described by Buchmann ${ }^{16)}$ ). This uncertainty is far to large for a rate of this importance. Based on nucleosynthesis arguments, a total S-factor of $170 \pm 20 \mathrm{keV} \mathrm{b}$ co-produces nearly all stable species between oxygen and calcium. ${ }^{19)}$ Uncertain stellar physics (semi-convection, and mass loss), also affects the total carbon and oxygen content in stars, but the current nuclear physics uncertainty is in utmost need of diminishment. Further experimental effort is highly encouraged.

\section{Neutron Sources and Sinks}

Secondary to energy generation, but no less important to nucleosynthesis, are the reactions that operate during helium burning to control the neutron budget. They directly affect the production of many species, most importantly those above the iron group made via the $s$-process. In massive stars the ${ }^{22} \mathrm{Ne}(\alpha, n)^{25} \mathrm{Mg}$ reaction, (in close competition with $\left.{ }^{22} \mathrm{Ne}(\alpha, \gamma){ }^{26} \mathrm{Mg}\right)$, provide the neutrons required to synthesize stable species with $60 \leq \mathrm{A} \leq 88$, or the so-called "weakcomponent" of the $s$-process. ${ }^{20)}$ The ${ }^{22} \mathrm{Ne}$ is produced by two alpha-captures on ${ }^{14} \mathrm{~N}$ produced in the hydrogen burning $\mathrm{CNO}$ cycle. These reactions operate predominantly during core helium burning, although they also operate (supplemented by ${ }^{26} \operatorname{Mg}(\alpha, n){ }^{29} \mathrm{Si}$ ) at later times in shell helium burning. The bulk of the $s$-process nuclei (with $\mathrm{A} \geq 88$ ) are synthesized during shell helium burning in low mass AGB stars, where the principle neutron source reaction is ${ }^{13} \mathrm{C}(\alpha, n){ }^{16} \mathrm{O}$.

The status of both ${ }^{22} \mathrm{Ne}$ alpha-capture reactions is highly

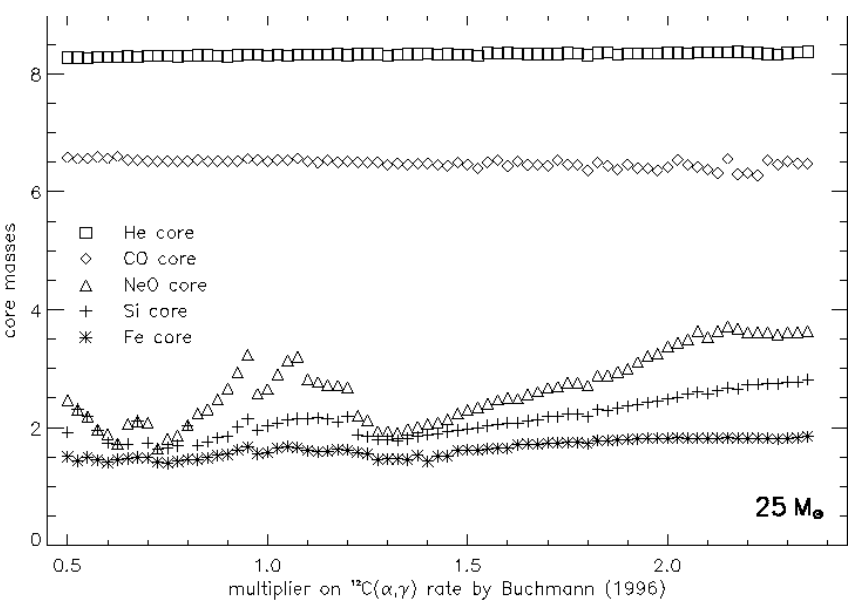

Fig. 2 Helium (squares), carbon-oxygen (diamonds), neon-oxygen (triangles), silicon (crosses), and "iron" (asterisks) core masses as a function of a multiplier on the ${ }^{12} \mathrm{C}(\alpha, \gamma){ }^{16} \mathrm{O}$ reaction rate of Buchmann. ${ }^{16)}$

uncertain owing to the large experimental difficulty in determining the strength of the $633 \mathrm{keV}$ resonance in the ${ }^{22} \mathrm{Ne}+\alpha$ channel. ${ }^{21)}$ Various choices for the parameters of this resonance give quite different strengths for the neutron exposure in the $s$-process, though none so powerful as to move the $s$-process peak much above $\mathrm{A}=90$. Recent studies ${ }^{22}$ suggest a diminished role for this reaction rate to a level no higher than the "lower-bound" recommended by Käppeler. ${ }^{21)}$ Figure 3 shows the sensitivity of the nucleosynthesis to variations in the ${ }^{22} \mathrm{Ne}(\alpha, n)^{25} \mathrm{Mg}$ rate that arise using the same stellar model that produced the results of Figure 1.

The current sources of these and other important $(\alpha, n)$ and $(\alpha, \gamma)$ reactions on targets lighter than Si are drawn from many experimental efforts. ${ }^{20)}$ Compilations do exist, ${ }^{14,15)}$ but the current uncertainties warrant further experiment, especially for the reactions involving ${ }^{18} \mathrm{O}$ and ${ }^{22} \mathrm{Ne}$.

In addition to the neutron source reactions, neutron capture reactions on all targets heavier than sodium are required to predict the $s$-process abundances in low and high mass stars. Fortunately, the reaction rate situation here is well in hand, in that nearly all of the important cross sections are measured in the temperature range of interest $(\sim 25-100 \mathrm{keV})$. Various compilations are available. ${ }^{23)}$ There are however many important $(n, \gamma)$ reactions on unstable targets that affect $s$-process branching which must be calculated by theory. ${ }^{3)}$

Finally, the nucleus ${ }^{60} \mathrm{Fe}$, a potential candidate for gammaline detection, is also made during helium burning in massive stars. Its synthesis is currently dependent on theoretical cross sections. $^{24)}$ RIA measurements that provide either cross section or level density information pertaining to the $(n, \gamma)$ rates on ${ }^{59,60} \mathrm{Fe}$ would be most welcome.

\section{Charged-particle Reactions on $\mathbf{Z} \leq 14$}

Reactions involving protons $[(p, \gamma),(\alpha, p),(p, \alpha)]$ and nuclei lighter than silicon are also important, especially those that are involved in the energy generation cycles that operate 

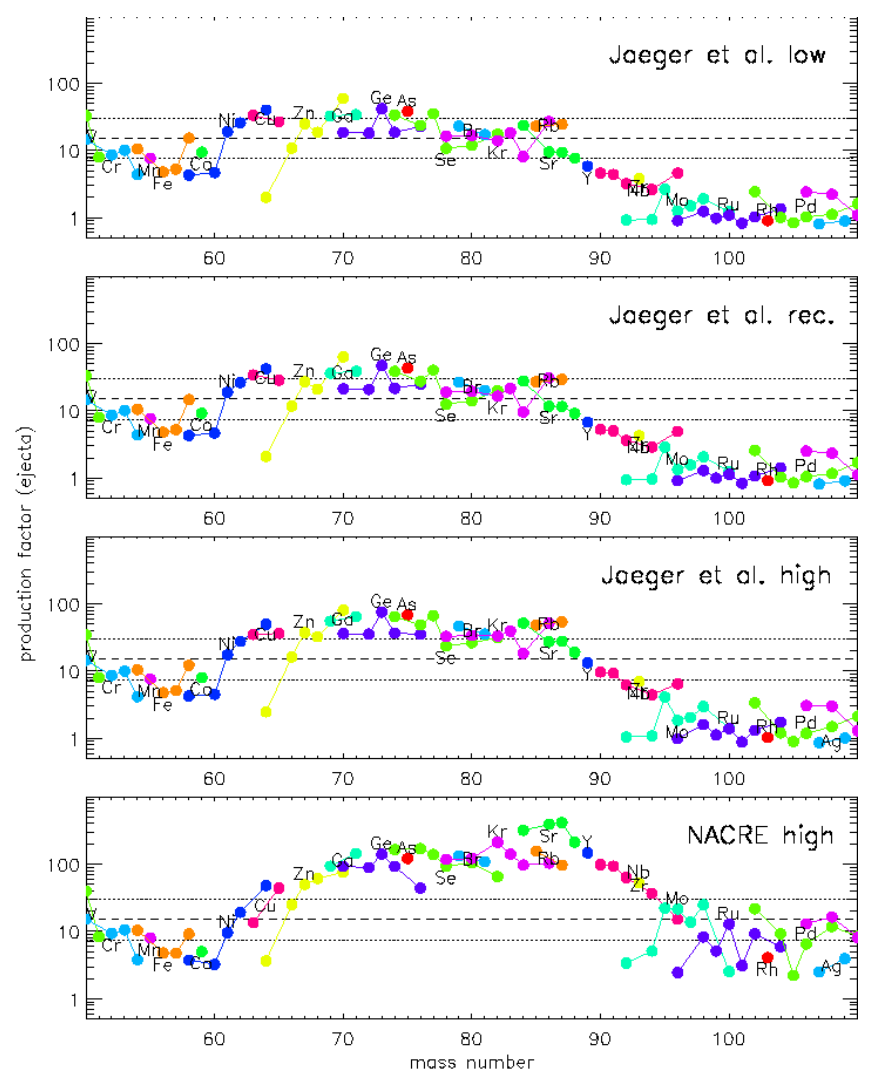

Fig. 3 Sensitivity of the production factors for different ${ }^{22} \mathrm{Ne}(\alpha, n){ }^{25} \mathrm{Mg}$ rates in a $25 \mathrm{M}_{\odot}$ star. The first three panels give the results for the lower limit, recommended value, and upper limit of Jaeger et al. ${ }^{22)}$ The bottom panel uses the evaluated reaction rate set from $\mathrm{NACRE}^{15)}$ with their upper limit for the ${ }^{22} \mathrm{Ne}(\alpha, n)^{25} \mathrm{Mg}$ rate.

during hydrogen burning (PPI, PPII, and PPIII in low mass stars; the CNO cycle and its bi-cycles in massive stars). Such reactions determine the synthesis of the important species ${ }^{13} \mathrm{C},{ }^{14,15} \mathrm{~N},{ }^{17,18} \mathrm{O},{ }^{21} \mathrm{Ne},{ }^{23} \mathrm{Na}$, and especially ${ }^{26} \mathrm{Al}$ (observed via $\gamma$-line decay). Of these low-mass stars make most of the ${ }^{13} \mathrm{C},{ }^{14} \mathrm{~N}$, and some of the ${ }^{23} \mathrm{Na}$ (through incomplete $\mathrm{CNO}$ processing and the Ne-Na cycle). It is estimated ${ }^{11)}$ that massive stars produce about one-fifth of the ${ }^{14} \mathrm{~N}$ in the sun. The production of ${ }^{15} \mathrm{~N}$ is relegated to novae. Controversy currently exists on how much of the observed $2 \mathrm{M}_{\odot}$ of ${ }^{26} \mathrm{Al}$ present in the Galaxy ${ }^{25)}$ is attributed to either massive stars, ${ }^{26,27)}$ or novae, ${ }^{28)}$ although massive stars can easily account for all of it. ${ }^{11)}$ Until recent revisions to the key reaction rates affecting ${ }^{17} \mathrm{O}$ synthesis, ${ }^{29)}$ massive stars were thought to be able to produce the solar abundance. ${ }^{11)}$ Should these new measurements be confirmed, production of ${ }^{17} \mathrm{O}$ may be relegated to low mass stars or novae. ${ }^{30)}$ Proton capture reactions on targets with $N \leq Z$ are also crucial for break-out of the hot-CNO cycle in $\mathrm{x}$-ray bursts.

Sources for these reaction rates are varied. ${ }^{14,15,31)} \mathrm{Al}$ though the reactions involving energy generation are adequately determined, ${ }^{32)}$ further experiment is needed.

\section{Charged-particle Reactions on $Z>14$}

The nucleosynthesis of nearly all species from silicon to the iron group are produced in the latter stages of stellar burning in massive stars. Many are made in either partial or complete nuclear statistical equilibrium, ${ }^{33)}$ where the absolute values of individual reaction rates are not as important as Qvalues and partition functions. ${ }^{24)}$ But for the $p$-process nuclei (Figure 1), accurate rates are required to predict the nucleosynthesis of these rare species.

Current nucleosynthesis calculations rely heavily on theoretical model calculations for nearly all of these cross sections. ${ }^{3)}$ This is troubling for some critical $(\alpha, \gamma),(\alpha, p)$, and $(p, \gamma)$ reactions on self-conjugate nuclei, which are difficult to measure (and predict) due to suppression of the photon transmission function due to uncertain isospin mixing. ${ }^{34)}$ Further problems exist due to the use of a poorly known global optical model $\alpha$-particle potential. This area is in desperate need of experimental work that either measures the most important cross sections or derives important structure data for use in calibrating the Hauser-Feshbach reaction models, especially for targets with $\mathrm{A}>60$.

\section{Weak Interaction Rates}

\section{Ground State Weak Rates}

Prior to carbon-burning, the use of ground state decay rates calculated from measured laboratory half-lives and branching ratios are adequate (in most instances) to predict stellar nucleosynthesis, including the $s$-process. These are the default source of weak reaction data for all species in modern reaction networks, ${ }^{35)}$ and are currently the only source of data beyond $A \sim 60$.

Of particular interest are the weak decay rates that affect the production of gamma-line species, particularly ${ }^{22} \mathrm{Na}(2.6$ yr), ${ }^{26} \mathrm{Al}\left(7.5 \times 10^{5} \mathrm{yr}\right),{ }^{44} \mathrm{Ti}(60 \mathrm{yr}),{ }^{56,57} \mathrm{Ni}(6.1 \mathrm{~d}, 1.5 \mathrm{~d})$, ${ }^{56,57,60} \mathrm{Co}(77.1 \mathrm{~d}, 271 \mathrm{~d}, 5.27 \mathrm{yr})$, and ${ }^{60} \mathrm{Fe}\left(1.5 \times 10^{5} \mathrm{yr}\right)$. Of these ${ }^{26} \mathrm{Al}$ has been observed in the Galatic plane and in individual supernova remnants. ${ }^{27)}$ The shorter lived ${ }^{44} \mathrm{Ti}$ has been observed in $\mathrm{Cas}^{25}$ ) and the $\mathrm{Ti}, \mathrm{Ni}$, and Co isotopes in SN 1987A. ${ }^{25)}$ Both ${ }^{22} \mathrm{Na}$ and ${ }^{60} \mathrm{Fe}$ are principle candidates for detection by the upcoming INTEGRAL mission. The lifetime for ${ }^{44} \mathrm{Ti}$ to decay to ${ }^{44} \mathrm{Ca}$ has been a subject of much experimental uncertainty over the last thirty years, with a half-life that has steadily increased from 45 to $70 \mathrm{yr}$. The most recent measurements seem to be converging on the presently accepted value of $60 \mathrm{yr}^{36)}$

\section{Temperature Dependent Weak Rates}

For the extreme conditions encountered in stellar collapse, temperature and density dependent weak rates ${ }^{37,38)}$ are required. These play a central role in determining the nucleosynthesis of many important $\gamma$-line species $\left({ }^{56,57} \mathrm{Ni},{ }^{44} \mathrm{Ti}\right)$, the stellar structure after oxygen burning, the electron mole number $\left(Y_{e}\right)$, and in determining the requisite conditions for an $r$-process to occur in the $\nu$-wind of Type II supernova cores. ${ }^{42)}$

Some important changes in the stellar models are due to recently revised weak rates, ${ }^{38)}$ the largest changes become ap- 
parent during core silicon burning and thereafter. Typically, they lead to an increase of the central $Y_{e}$ (the electron mole number, or number of electrons per baryon) at the onset of core collapse by 2 to $3 \%$ and this difference tends to increase with increasing stellar mass. ${ }^{39)}$ Perhaps more important for the explosion mechanism of core collapse supernovae is an increase of the density in the mass range of $m=1.5 \mathrm{M}_{\odot}$ to 2 $\mathrm{M}_{\odot}$ by $30-50 \%$ relative to the same models computed with the previous set of weak rates. ${ }^{10)}$ This may significantly affect the dynamics of the core collapse. The evolution of the central $Y_{e}$, the net weak flow, and their sensitivity to the choice of weak reaction rates is presented in (Figure 4) for a $15 \mathrm{M}_{\odot}$ star.

\section{Neutrino Loss Rates}

Neutrino losses are a critical aspect of stellar evolution in massive stars beginning with carbon burning. The dominant losses before silicon burning are due to thermal processes (chiefly pair-annihilation), which provide a loss term that is very roughly proportional to $\mathrm{T}^{9}$ in the range of interest for advanced burning stages. ${ }^{40)}$ This temperature sensitivity, combined with the need to burn heavier fuels at higher temperatures to surmount the increasing charge barriers, is what leads to the rapid decrease in lifetime to burn a given fuel reflected in (Figure 1), with obvious consequences for nucleosynthesis. The theory is well developed. ${ }^{41)}$

\section{Neutrino-interaction Rates and Branching Ratios}

Following Type II core collapse, a nascent neutron star liberates its gravitational binding energy $\left(\sim 3 \times 10^{53} \mathrm{erg}\right)$ over a Kelvin-Helmholtz contraction time scale $(\sim 10 \mathrm{~s})$ via neutrino emission, providing for the passage of a huge flux of neutrinos through the overlying shells of matter. This can cause an interesting transmutation of several rare elements by exciting abundant species to particle unbound levels through neutral current neutrino scattering. ${ }^{7)}$ Based on an extensive grid of supernova models ${ }^{10)}$ and a survey of galactic chemical evolution, ${ }^{11)}$ the most affected species are ${ }^{7} \mathrm{Li},{ }^{11} \mathrm{~B}$, and ${ }^{19} \mathrm{~F}$. Several other species $\left({ }^{15} \mathrm{~N},{ }^{26} \mathrm{Al}\right.$, and the rarest naturally occurring elements ${ }^{138} \mathrm{La}$ and ${ }^{180} \mathrm{Ta}$ ) show modest enhancement over their production via more conventional nucleosynthetic processes. Neutral and charged current neutrino interaction cross sections and branching ratios are calculated from theory. ${ }^{7,43)}$

\section{Conclusion}

The field of nucleosynthesis has come a long way since the seminal papers of $1957,{ }^{4)}$ but certain key nuclear quantities still have unacceptably large uncertainties. Chief among them are the reaction rates for ${ }^{12} \mathrm{C}(\alpha, \gamma){ }^{16} \mathrm{O}$ and ${ }^{22} \mathrm{Ne}(\alpha, n){ }^{25} \mathrm{Mg}$. Much experimental work has been done to improve the situation, ${ }^{14,15,31)}$ but much more remains to be done. A major challenge will be to reduce the previously acceptable "factor of two" accuracy ascribed to reaction rates derived from nuclear modeling. ${ }^{24)}$ Such goals are worthy in light of the wealth of new and highly accurate observational data from a variety of astrophysical systems.

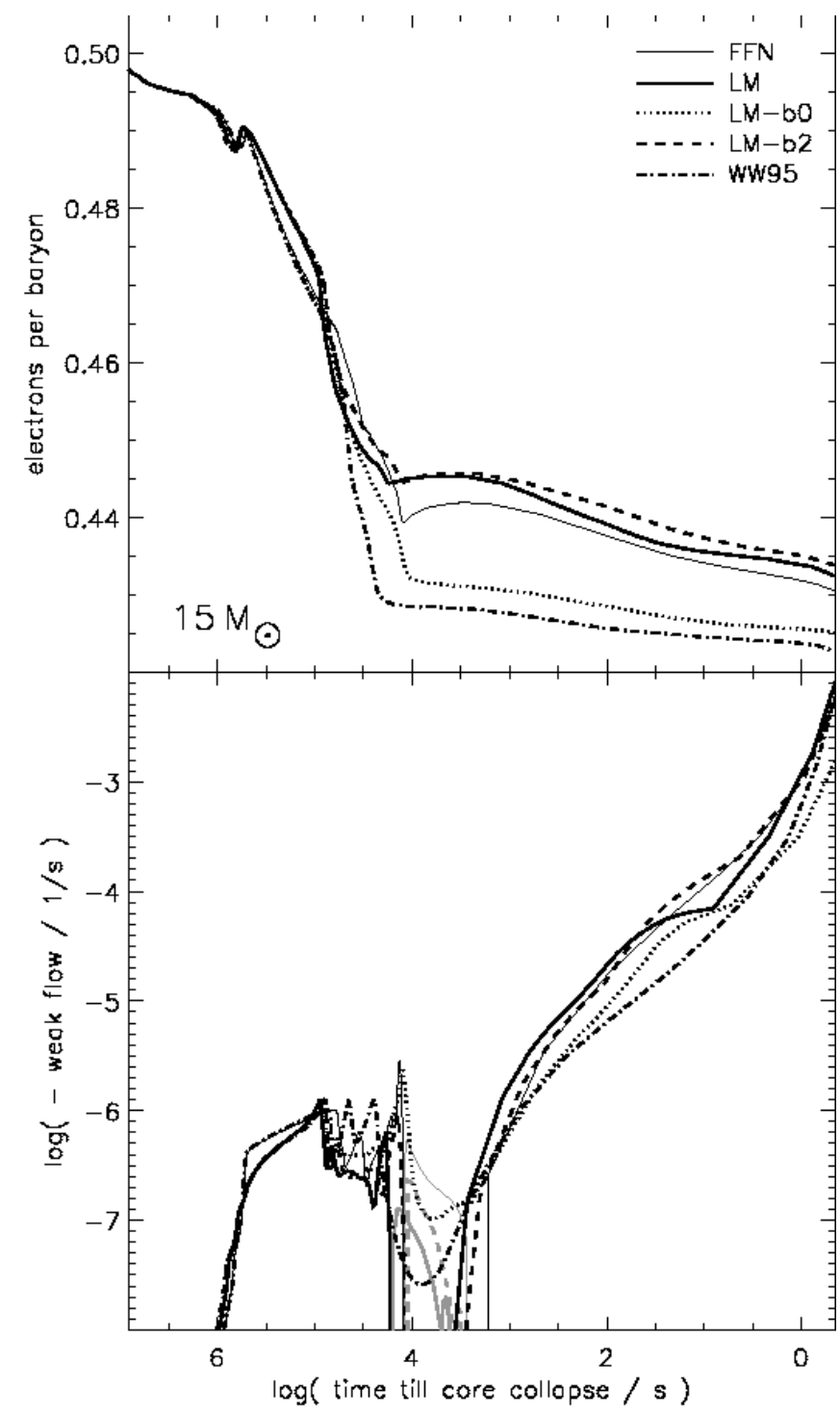

Fig. 4 Evolution of $Y_{e}$ and the net weak flow in the center of a 15 $\mathrm{M}_{\odot}$ star followed from central oxygen depletion till the onset of core collapse. Five choices of weak interaction rate sets are used, FFN ${ }^{37)}$ (the previous standard), and three sets from Langanke \& Martinez-Pinedo. ${ }^{38)}$ LMP is the recommended set, the $-\mathrm{b} 0$ and $-\mathrm{b} 2$ lines indicate rate sets that multiplied the recommended beta-decay rates by zero and two respectively. The last simulation (WW95) is the result from a previous stellar model ${ }^{10)}$ that used FFN rates.

\section{Acknowledgment}

This work was performed under the auspices of the U.S. Department of Energy by the University of California Lawrence Livermore National Laboratory under contract No. W-7405-Eng-48. S. E. Woosley acknowledges support from the DOE ASCI Program and the National Science Foundation (AST-97-31569). T. Rauscher acknowledges support by a PROFILE professorship from the Swiss National Science Foundation (grant 2124-055832.98). 


\section{References}

1) S. E. Woosley, W. A. Fowler, J. A. Holmes, B. A. Zimmerman, "Semiemperical thermonuclear reaction-rate data for intermediate-mass nuclei," At. Data Nucl. Data Tables, 22, 371 (1978).

2) F.-K. Thielemann, M. Arnould, J. Truran, in Advances in $\mathrm{Nu}$ clear Astrophysics, p. 525, (1987).

3) T. Rauscher, F.-K. Thielemann, "Astrophysical reaction rates from statistical model calculations," At. Data Nucl. Data Tables, 75, 1, (2000).

4) E. M. Burbidge, G. R. Burbidge, W. A. Fowler, F. Hoyle, "Synthesis of the elements in stars," Rev. Mod. Phys., 29, 547 (1957).

5) G. Wallerstein et al., "Synthesis of the elements in stars, forty years of progress," Rev. Mod. Phys., 69, 995 (1997).

6) S. E. Woosley, A. Heger, T. Weaver, "The evolution and explosion of massive stars," Ann. Rev. Astron. \& Astrophys., in press (2002).

7) S. E. Woosley, D. H. Hartmann, R. D. Hoffman, W. C. Haxton, "The $\nu$-Process," Astro. Phys. Journal 356, 272 (1990).

8) S. E. Woosley, M. Howard, "The $p$-Process in supernovae," Astro. Phys. Journal Suppl., 36, 285 (1978).

9) S. E. Woosley, R. D. Hoffman, "The $\alpha$-Process and the $r$-Process," Astro. Phys. Journal, 395, 202 (1992).

10) S. E. Woosley, T. A. Weaver, "The evolution and explosion of massive stars II. Explosive hydrodynamics and nucleosynthesis," Astro. Phys. Journal Suppl., 101, 181 (1995).

11) F. X. Timmes, S. E. Woosley, T. A. Weaver, "Galactic chemical evolution: hydrogen through zinc," Astro. Phys. Journal Suppl., 98, 617 (1995).

12) T. Rauscher, A. Heger, S. E. Woosley, R. D. Hoffman, "Nucleosynthesis in massive stars with improved nuclear and stellar physics," Astro. Phys. Journal, in preparation (2002).

13) A. Heger, R. D. Hoffman, T. Rauscher, S. E. Woosley, "Nucleosynthesis in massive stars including all stable isotopes," Proc. $10^{\text {th }}$ workshop on "Nuclear Astrophysics", MPA-P12, p. 105 (2000).

14) G. A. Caughlan, W. A. Fowler, "Thermonuclear reaction rates V,” At. Data Nucl. Data Tables, 40, 283 (1988).

15) C. Angulo et al., "A compilation of charged-particle induced thermonuclear reaction rates," Nucl. Phys., A656, 3 (1999).

16) L. Buchmann, "New Stellar Reaction Rate for ${ }^{12} \mathrm{C}(\alpha, \gamma){ }^{16} \mathrm{O}$," Astro. Phys. Journal, 468, 127 (1996).

17) R. E. Azuma et al., "Constraints on the low-energy E1 cross section of ${ }^{12} \mathrm{C}(\alpha, \gamma){ }^{16} \mathrm{O}$ from the $\beta$-delayed alpha-spectrum of ${ }^{16}$ N," Phys. Rev. C, 50, 1194 (1994).

18) R. Kunz et al., " ${ }^{12} \mathrm{C}(\alpha, \gamma){ }^{16} \mathrm{O}$, the key reaction in stellar nucleosynthesis," Phys. Rev. Lett., in press.

19) T.A. Weaver, S.E. Woosley, "Nucleosynthesis in massive stars and the ${ }^{12} \mathrm{C}(\alpha, \gamma){ }^{16} \mathrm{O}$ reaction rate," Phys. Rep., 227, 65 (1993).

20) R. D. Hoffman, S. E. Woosley, T. A. Weaver, "Nucleosynthesis below $A=100$ in massive stars," Astro. Phys. Journal, 549, 1085 (2001).

21) F. Käppeler, et al., "Reaction rates for ${ }^{18} \mathrm{O}(\alpha, \gamma){ }^{22} \mathrm{Ne}$, ${ }^{22} \mathrm{Ne}(\alpha, \gamma){ }^{26} \mathrm{Mg}$, and ${ }^{22} \mathrm{Ne}(\alpha, n){ }^{25} \mathrm{Mg}$ in stellar helium burning and s-process nucleosynthesis in massive stars," Astro. Phys. Journal, 437, 396 (1994).

22) M. Jaeger, R. Kunz, A. Mayer, J. W. Hammer, J. W. Staudt, K.L. Kratz, B. Pfeiffer, " ${ }^{22} \mathrm{Ne}(\alpha, n)^{26} \mathrm{Mg}$, the key neutron source in stars," Phys. Rev. Let., in press (2001).

23) Z. Y. Bao, H. Beer, F. Käppeler, F. Voss, K. Wisshak, T. Rauscher, "Neutron cross sections for nucleosynthesis studies," At. Data Nucl. Data Tables, 76, 70 (2000).
24) R. D. Hoffman, S. E. Woosley, T. A. Weaver, T. Rauscher, F.-K. Thielemann, "The reaction rate sensitivity of nucleosynthesis in type II supernovae,” Astro. Phys. Journal, 521, 735 (1999).

25) R. Diehl, F. X. Timmes, "Gamma-ray line emission from radioactive isotopes in stars and galaxies," Proc. Astron. Soc. Pacific, 110, 637 (1998).

26) G. Meynet, M. Arnould, N. Prantzos, G. Paulus, "Contribution of wolf-rayet stars to the synthesis of ${ }^{26} \mathrm{Al}$. I. The $\gamma$-ray connection," Astro. \& Astrophys., 320, 460 (1997).

27) F. X. Timmes, S. E. Woosley, D. H. Hartmann, R. D. Hoffman, T. A. Weaver, F. Matteucci, “26 $\mathrm{Al}$ and ${ }^{60} \mathrm{Fe}$ from supernovae explosions," Astro. Phys. Journal, 449, 204 (1995).

28) R. D. Gehrz, J. W. Truran, R. E. Williams, S. Starrfield, "Nucleosynthesis in classical novae and its contribution to the interstellar medium," Proc. Astron. Soc. Pacific, 110, 3 (1998).

29) V. Landré, N. Prantzos, P. Aguer, G. Bogaert, A. Lefebvre, J. Thibaud, "Revised reaction rates for the H-burning of ${ }^{17} \mathrm{O}$ and the oxygen isotopic abundances in red giants," Astro. \& Astrophys., 240, 85 (1990).

30) J. Jose, M. Hernanz, "Nucleosynthesis in classical novae: CO versus ONe white dwarfs," Astro. Phys. Journal, 494, 680 (1998).

31) C. Iliadis, J. M. D’ Auria, S. Starrfield, W. J. Thompson, M. Wiescher, "Proton-induced thermonuclear reaction rates for $\mathrm{A}=20$ 40 nuclei," Astro. Phys. Journal Suppl., 134, 151 (2001).

32) E. G. Adelberger et al., "Solar fusion cross sections", Rev. Mod. Phys., 70, \#4, 1265 (1998).

33) S.E. Woosley, D. Arnett, D. D. Clayton, "The explosive burning of oxygen and silicon," Astro. Phys. Journal Suppl., 26, 213 (1973).

34) T. Rauscher, F.-K. Thielemann, J. Görres, M. Wiescher, "Capture of $\alpha$-particles by isospin-symmetric nuclei," Nucl. Phys., A675, 695 (2000).

35) J. Tuli, "Nuclear Wallet Cards," National Nuclear Data Center, Brookhaven National Lab. (1990).

36) J. Görres et al., "Half-life of ${ }^{44} \mathrm{Ti}$ as a probe for supernova models," Phys. Rev. Lett., 80, \# 12, 2554 (1998).

37) G.M. Fuller, W.A. Fowler, M.J. Newman, "Stellar weak interaction rates for intermediate mass nuclei III. Rate tables for the free nucleons and nuclei with A=20 to A=60," Astro. Phys. Journal Suppl., 48, 279 (1982).

38) K.-H. Langanke, G. Martinez-Pinedo, "Shell-model calculations of stellar weak interaction rates: II. Weak rates for nuclei in the mass range $\mathrm{A}=45-65$ in supernovae environments," Nucl. Phys., A673, 481 (2000).

39) A. Heger, K.-H. Langanke, G. Martinez-Pinedo, S. E. Woosley, "Presupernova collapse models with improved weak-interaction rates," Phys. Rev. Lett., 86, \#9, 1678 (2001).

40) D. D. Clayton, Principles of stellar evolution and nucleosnythesis, (Univ. of Chicago Press, Chicago, 1983) p. 276.

41) N. Itoh, H. Hayashi, A. Nishikawa, Y. Kohyama, "Neutrino energy loss in stellar interiors. VII. Pair, photo-, plasma, bremsstrahlung, and recombination neutrino processes," Astro. Phys. Journal Suppl., 102, 411 (1996).

42) R. D. Hoffman, S. E. Woosley, Y.-Z. Qian, "Nucleosynthesis in neutrino-driven winds II. Implications for heavy element synthesis," Astro. Phys. Journal, 482, 951 (1997).

43) E. Kolbe, K.-H. Langanke, "Role of $\nu$-induced reactions on lead and iron in neutrino detectors" Phys. Rev. C, 63, \#2, 5802 (2001). 